\title{
HiRISE - High-Resolution Imaging and Spectroscopy Explorer - Ultrahigh resolution, interferometric and external occulting coronagraphic science
}

\author{
Robertus Erdélyi 1,6,16 (D) - Luc Damé ${ }^{2}$ - Andrzej Fludra ${ }^{3} \cdot$ Mihalis Mathioudakis $^{4}$. \\ T. Amari ${ }^{5} \cdot$ B. Belucz ${ }^{1,6}$, et al. [full author details at the end of the article]
}

Received: 7 August 2020 / Accepted: 27 December 2021 / Published online: 5 March 2022

(c) The Author(s) 2022

\begin{abstract}
Recent solar physics missions have shown the definite role of waves and magnetic fields deep in the inner corona, at the chromosphere-corona interface, where dramatic and physically dominant changes occur. HiRISE (High Resolution Imaging and Spectroscopy Explorer), the ambitious new generation ultra-high resolution, interferometric, and coronagraphic, solar physics mission, proposed in response to the ESA Voyage 2050 Call, would address these issues and provide the best-ever and most complete solar observatory, capable of ultra-high spatial, spectral, and temporal resolution observations of the solar atmosphere, from the photosphere to the corona, and of new insights of the solar interior from the core to the photosphere. HiRISE, at the L1 Lagrangian point, would provide meter class FUV imaging and spectroimaging, EUV and XUV imaging and spectroscopy, magnetic fields measurements, and ambitious and comprehensive coronagraphy by a remote external occulter (two satellites formation flying $375 \mathrm{~m}$ apart, with a coronagraph on a chaser satellite). This major and state-of-the-art payload would allow us to characterize temperatures, densities, and velocities in the solar upper chromosphere, transition zone, and inner corona with, in particular, $2 \mathrm{D}$ very high resolution multi-spectral imaging-spectroscopy, and, direct coronal magnetic field measurement, thus providing a unique set of tools to understand the structure and onset of coronal heating. HiRISE's objectives are natural complements to the Parker Solar Probe and Solar Orbiter-type missions. We present the science case for HiRISE which will address: i) the fine structure of the chromosphere-corona interface by 2D spectroscopy in FUV at very high resolution; ii) coronal heating roots in the inner corona by ambitious externally-occulted coronagraphy; iii) resolved and global helioseismology thanks to continuity and stability of observing at the L1 Lagrange point; and iv) solar variability and space climate with, in addition, a global comprehensive view of UV variability.
\end{abstract}

Keywords Solar physics mission · Ultra-high resolution · Interferometry · Coronagraph $\cdot$ Solar atmosphere $\cdot$ Photosphere $\cdot$ Chromosphere $\cdot$ Corona 


\section{Introduction}

Recent developments in solar physics have shown the definitive role of magnetic fields and waves at the chromosphere-corona interface and deep in the inner corona, where dramatic and physically dominant changes occur [e.g., 1, 5, 7, 24, $33,41,57]$. Advances in new-generation ultra-high spatial, temporal and spectral resolution, interferometry, and coronagraphy, now provide the opportunity to make transformational progress in addressing the finest details of these processes through observations of the solar atmosphere from the photosphere to the corona, leading to new insights of the solar interior from the core to the photosphere.

Despite excellent progress in recent years in understanding the link between the solar atmosphere, coronal dynamics, and coronal heating [e.g., 7, 23, 24, 31, $32,33,53,60,68]$, there remain major crucial questions that are still unanswered. Limitations in the observations are one important reason. Both theoretical and observational considerations point to the importance of small spatial scales of impulsive energy release, dominant dynamics, and extreme plasma non-uniformity [e.g., 1, 5, 7, 10, 20]. Consequently, high spatial resolution, broad temperature coverage with high temperature fidelity and sensitivity to velocities and densities, are all critical observational parameters. Current instruments lack one or more of these much-needed properties for major leaps forward. Recent novel developments in coronal heating and diagnostics emphasize that high spatial resolution observations, especially imaging spectroscopy and spectropolarimetry, are absolutely necessary to make major progress on this fundamental problem of modern astrophysics [e.g., 11, 16]. In particular, the critical region between the chromosphere and corona, the transition zone (or, in a broader context: the Interface Region), may need to be addressed as well as measuring the nature and connectivity of magnetic fields from the photosphere to the corona, directly.

There are four major thematic issues that address the global understanding of the solar environment through the magnetic field emergence and dissipation, and its influence on Earth:

1. Revealing and understanding the detailed structure and evolution of the solar atmosphere;

2. Answering longstanding and fundamental solar physics questions;

3. Probing the internal, sub-photospheric structure of the Sun, the transfer from the radiative to the convection zone, the role of the tachocline, the links between internal flows and the magnetic cycle.

4. Measuring and understanding the profound influence of the Sun on Earth and the consequences for human life through the predictions of long-term climatic changes.

Theses themes are interlinked and also complementary: the internal structure of the Sun determines the surface activity and dynamics that trigger magnetic field structuring, of which evolution, variation, and dissipation will, in turn, explain 

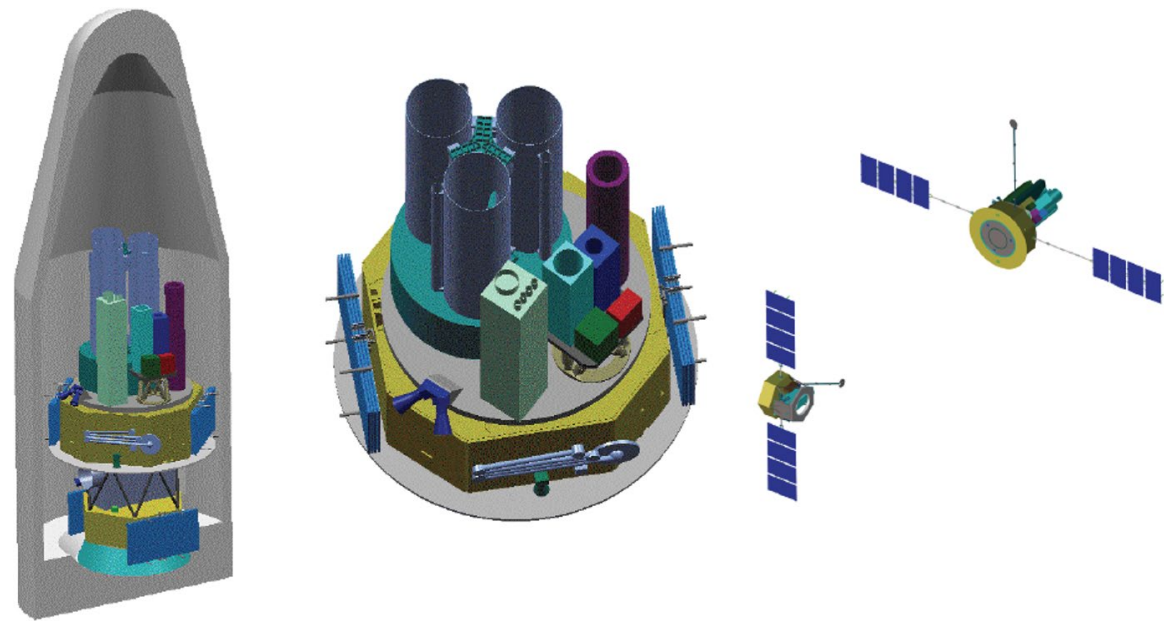

Fig. 1 Schematic illustration of the two satellites of the HiRISE mission in stacked position in the SoyuzFregat fairing (left); Details of the possible accommodation of the HiRISE model payload on the main satellite (Herschel platform), middle; The two satellites, main and chaser, of HiRISE in formation flying configuration $375 \mathrm{~m}$ apart (right). The figure is an evolution from the initial Cosmic Vision proposal in June 2007, followed by various later mission concepts, including e.g. M3 (2010), M4 (2015) and M5 (2016)

the coronal heating onset and the major energy releases that feed the influence of the Sun on Earth.

To make progress in these thematic areas we propose a concept for HiRISE (High-Resolution Imaging and Spectroscopy Explorer), an ultra-high resolution, interferometric, and coronagraphic, solar physics mission. Situated at the L1 Lagrangian point, HiRISE would provide meter class FUV imaging and spectro-imaging, EUV and XUV imaging and spectroscopy, magnetic fields measurements, and ambitious coronagraphy by a remote external occulter (two satellites formation flying $375 \mathrm{~m}$ apart, with a coronagraph on a chaser satellite, see Fig. 1). This major and state-of-the-art payload will allow characterization of temperature, densities, and velocities in the solar upper chromosphere, transition zone, and inner corona with, in particular, $2 \mathrm{D}$ very high resolution multi-spectral imaging-spectroscopy and direct coronal magnetic field measurement, thus providing a unique set of tools to understand the structure and onset of coronal heating. The scientific objectives are natural complements to the Solar Orbiter and Parker Solar Probe-type missions that lack duty cycle, high resolution, spatial, spectral, and temporal multi-temperature diagnostics and full coronal magnetometry.

In summary, this was a White Paper, submitted in response to the ESA Voyage 2050 Call [see 65]. After the above Introduction, the next section is addressing the scientific objectives. An outline of the scientific requirements can be found in Section 3. A model payload and mission profile are presented in Sections 4 and 5 , respectively. Section 6 contains some remarks on International Collaboration. 


\section{Detailed science objectives}

\subsection{Context}

The "Big" science questions in Solar Physics are:

- the interface heating process; discriminate between theories of wave/impulsive plasma heating

- how and where the solar wind is accelerated

- the nature of solar flares and CME's

- the origin of the sunspot cycle (the variability)

- the nature of the solar interior

These questions have to be directly addressed in the near future, since both the atmospheric heating and acceleration process (or processes) and the nature of solar flares are linked with the magnetic field which is the key to understanding these dissipation mechanisms since they necessarily involve small scales. Helioseismology of the deep solar interior is a problem that requires the detection of long-period modes and, if accessible, $g$-modes, the internal gravity modes. Since $g$-modes are probably best observed at the limb, as indicated by MDI [37], and in the UV (theory predicts about two orders of magnitude better sensitivity with measuring displacement, like the diameter of the Sun, than with measuring intensity or velocity on disc), a dedicated instrument is devoted to those measurements (SUAVE). The origin of the solar cycle is a variability problem and an important issue for life on Earth. We need to investigate the consequences of solar variations (constant, UV, diameter) on the Earth climate or, on shorter time scales, for Space Weather issues.

All these questions must be addressed by HiRISE which will provide new views, diagnostics and give answers to those not addressed by the in situ plasma missions such as: Parker Solar Probe, Solar Orbiter and/or InterHelioProbe. HiRISE will deliver well with its high-resolution of the magnetic structuring, coronal diagnostics, magneto-helioseismology for solar interior (dynamo, $g$-modes), coronal magnetic field, Lyman- $\alpha$, solar shape and solar variability for Space Weather and Space Climate.

Missions such as SOHO, Hinode, STEREO, RHESSI, SDO, and IRIS have been very successful in addressing many aspects of solar and heliospheric physics and, consequently, pointing out the areas in need of a better and deeper understanding of the Sun and the heliosphere. They were truly worthy missions; but now progress can be achieved in areas where SOHO and other missions stopped: high imaging, temporal, and spectroscopic resolution of the surface and atmosphere, coronagraphy of the inner corona with a direct measurement of the magnetic field, and global and local optimized magneto-helioseismology. The series of recent missions, simply do not have the resolution (in particular at the crucial interface between the chromosphere and corona) nor the coronagraphs to address one of the most enduring enigmas in astrophysics: the existence of a million degree solar corona. 
Ultra-high resolution measurement is a further development of the SIMURIS, SPI, and SOLARNET concepts $[11,15,16]$. HiRISE is a significant step further than any other proposed mission by implementing a global concept that delivers not only extreme solar coronagraphy (direct coronal magnetic field measurement), but also the very high spatial and spectral resolutions in the optically thin UV, FUV, and EUV lines required to understand the confinement in the transition zone, the interface between the chromosphere and corona. It includes high resolution and performing EUV/XUV imaging and spectroscopy, very high resolution made possible by interferometry, and adds a helioseismology package with an enhanced resolved velocity experiment (the DMOF: Dual Magneto Optical Filter) and a multi-point global velocity measure (GOLF-NG, see e.g. [64]), that could potentially address the so-much sought-after $g$-modes detection.

Altogether, our science and the associated enabling mission is now proposing further improved, innovative, European and international (China, US, and/or Russia) instruments, building upon our previous responses to ESA for M2 (2007) [12], M3 (2010), and M4 (2015) with the HiRISE Mission, notably with considerable overlaps in 2010 by the follow-up SolMex proposal [52]. HiRISE's extensive instrumentation covers all the required scientific objectives for an in-depth understanding of solar physics.

\subsection{Major scientific themes}

The global understanding of the solar environment through the magnetic field emergence and dissipation, and its influence on Earth, is at the centre of the four major themes addressed by HiRISE. These are:

1. to reveal and understand the detailed structure and evolution of the solar atmosphere. Ultra-high resolution imaging and spectroscopy will trace the Sun's magnetic field structure and evolution from the deep photosphere to the corona. It will reveal the links between the building layers of the Sun's atmosphere. We need to track the complete evolution of magnetically driven processes from the smallest scales to the largest, address magnetic emergence, evolution, and reconnection, the development and regression of active regions, the development and nature of transient events in the magnetised atmosphere, the onset and fine structure of flares from the smallest (e.g. nanoflare scales) to the largest (white light flares), and the propagation of magnetic activity through the different regimes of the solar atmosphere.

2. to answer longstanding and fundamental solar physics questions: How is the solar atmosphere heated? How does magnetic energy build upon the corona? What is the role of fine structure (strands)? Of convective motions? What is the role of waves, wave and/or ion-cyclotron, shock acceleration, dissipation, and energisation? How and where are the different components of the solar wind, slow and fast, accelerated? How are Coronal Mass Ejections accelerated? What is the strength of the coronal magnetic field? What is the magnetic building block of the corona and what is its topology, connectivity, and coupling role? With a unique 
set of coronagraphs, externally occulted for inner corona access, and ultraviolet for direct magnetic field measurement in the corona, HiRISE has the privilege to directly address these questions.

3. to evidence the internal, sub-photospheric structure of the Sun, the transfer from the radiative to the convection zone, the role of the tachocline, the links between internal flows and the magnetic cycle. Magnetic activity and its variations are the consequences of the internal regimes of convection. $g$-modes and long-period $p$-modes are the only known modes capable to penetrate deeply enough to constrain the solar dynamics of the core. These modes will be searched at the limb and in the UV displacements, where their amplitude is expected to be 4 to 5 times greater than with full-disc intensity or velocity measurements.

4. to measure and understand the profound influence of the Sun on Earth and the consequences for human life through the predictions of long-term climatic changes. For this, stratospheric UV and IR flux inputs, Lyman- $\alpha$ imaging, and in-situ measurements of the solar wind at L1 are needed to measure the solar shape, solar constant, and solar spectral irradiance variability. They are necessary to measure and follow in great detail the solar inputs received on Earth and their local and global variations with high precision.

We can reformulate these four themes in the context of HiRISE as:

1: Fine structure of the chromosphere-corona interface. The most interesting and novel observations will be made by a cophased interferometer of 1.4-meter baseline coupled to a UV double monochromator and Imaging Fourier Transform Interferometer to join high spatial, temporal, and spectral resolutions, to a visible 8-channels Vector Magnetograph (VM), and to a chromospheric imager. Using interferometry in the UV with a spectro-imaging mode will allow remote sensing of the solar surface and atmosphere with an unprecedented spatial resolution of $20 \mathrm{~km}$ on the Sun that current missions, despite their closer distance to the Sun, will never be able to achieve. High resolution EUV and X-ray imagers and spectrometers complete the view with both high resolution context views and detailed (to a tenth of an arcsec) in-depth imaging.

2: Atmospheric Heating. The problem of plasma heating roots in the inner corona has been around for many decades and progress has been made. It is now known that the origin of this once mysterious heating is most probably magnetic in nature. However, one still needs to probe the magnetic field further with high spatial, spectral, temporal, and velocity resolution in order to distinguish between, for example, magnetic wave heating, small-scale reconnection events, or convective leakage. Furthermore, the nature of the magnetic field needs to be accurately understood in order to construct a clear scenario of the magnetic building blocks of the solar atmosphere. Connectivity is a key ingredient that is poorly understood at present. Different phenomena, such as active regions, "Quiet Sun", and coronal holes are all likely to have different heating mechanisms. One needs now to determine the magnetic field with very high resolution in the transition zone and with e.g. a giant externally occulted coronagraph (formation flying) in the visible, IR, and UV, with direct measurement of the coronal magnetic field very near the limb (1.01 solar radii). 
3: Ambitious Magneto-Helioseismology. A permanent Sun-viewing orbit, like that of SOHO or SDO, allows resolved and global helioseismology, and magnetoseismology. This is the third breakthrough that is much needed for science progress: measuring limb oscillations in the UV (predicted to provide the highest $g$-modes sensitivity), intensity, global, and resolved observations, and high-resolution full and resolved Sun velocity oscillations (with e.g. the Dual Magneto Optical Filter (DMOF).

4: Solar Variability and Space Climate. The fourth breakthrough is the thorough set of diagnostics to study solar variability. A complete new set of solar constant, global, and spectrally resolved irradiance monitors, are implemented and coupled to enhanced Lyman- $\alpha$ imaging and a unique solar limb shape (and solar differential rotation) monitor. To this fourth set of goals, potential instruments like the VM and DMOF would bring the magnetic field information, and the coronagraphs, the coronal extension of the field. This will directly address the nature of UV variability and its climate consequences. EUV, X-ray imagers, and X-ray and gamma-ray spectrometers complete the variability survey as well as in-situ measurements of the solar wind at L1 (particles and magnetic field) that allow the development of interplanetary perturbations to be monitored, and their geoeffectiveness to be studied.

The high-resolution imaging of the solar atmosphere will be better than in past missions by more than an order of magnitude. These capabilities in concert will enable us to analyze thoroughly the time-variability, evolution, and fine-scale structure of the dynamic chromosphere-corona interface, to study fully the Sun's magnetic activity on multiple scales, to investigate energetic particle acceleration, confinement, and release, and to reveal plasma and radiation processes underlying the heating of the chromosphere and corona.

The relevant minimum observable scale in the solar atmosphere may be of the order of 10-30 km since smaller scales will probably be smeared out by plasma micro-instabilities (such as drift waves). This scale range is comparable to the photon mean free path in the chromosphere. Slightly larger scales can be expected in the corona (though the gradient across coronal loops may also be a few km). Altogether this situation is rather fortunate because we have access to higher resolutions in the far UV and EUV than in the visible. In the EUV and UV, the emission lines are generally optically thin, i.e. not affected by the optically thick radiative transfer conditions (which prevail in the visible and near UV lines accessible from ground or used on Hinode) and we can expect to see structures with scales $10-30 \mathrm{~km}$. In the visible, optically thick radiation in the atmosphere blurs the signature of structures and nothing smaller than 70-100 km should be observed. This means that with a single instrument of meter class diameter we have the appropriate, scientifically justified, spatial resolution for both the far UV (20 km in the C III line $117.5 \mathrm{~nm})$ and the near UV (60 km in the Ca II K line $\lambda 396.3 \mathrm{~nm}$ ).

Furthermore, observations of the EUV spectral range provide detailed plasma diagnostics in the solar atmosphere across the broad temperature range from tens of thousands to several millions $\mathrm{K}$, covering the chromosphere, transition region, and active corona. Analysis of the emission lines from trace elements in the Sun's atmosphere provides information on plasma density, temperature, flow speeds, and the structure and evolution of atmospheric phenomena. A range of lines from 
Fig. 2 SOHO/SUMER observations of the solar-wind source regions and magnetic structure of the chromospheric network. The insert shows the measured Doppler-shifts of Neon ions, indicating blue shifts, i.e. outflow, at the network cell boundaries and lane junctions below the polar coronal hole, and red-shifts (down-flow) in the network regions underlying the globally closed corona (adapted from [30])

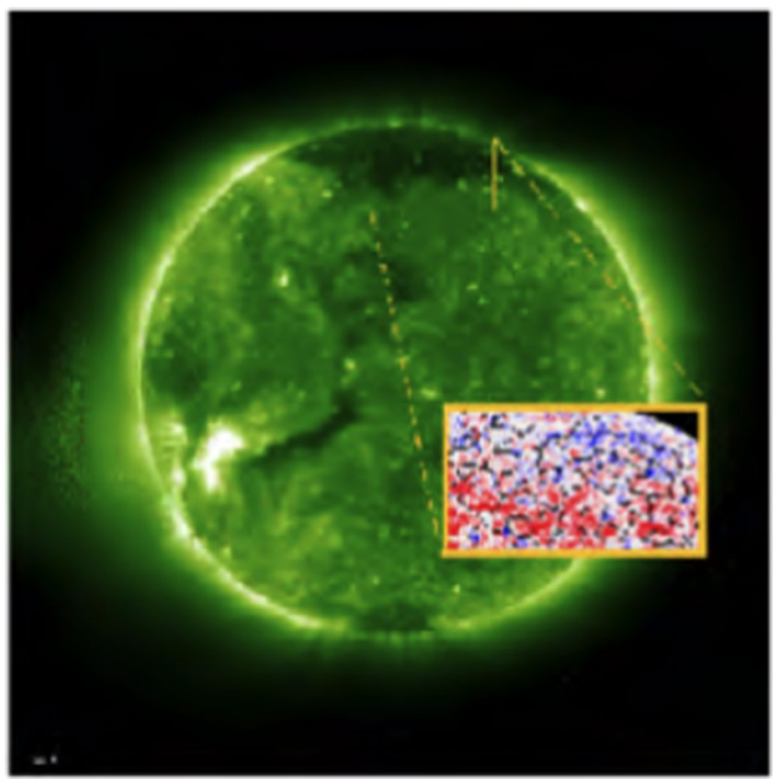

different elements provides a detailed element abundance diagnostic capability. In this respect, HiRISE ideally complements the in situ plasma studies that are carried by a solar probe.

A breakthrough in high spatial resolution observations in the UV (20 $\mathrm{km}$ is 20 times more spatial resolution than any previous solar instrument in space) should allow processes like magnetic heating in coronal loops (temperature profiles, time dependence, spatial local ionization of heating processes) to understood in finer physical details but, also, by access to visible wavelengths, the coupling between turbulent convective eddies and magnetic fields in the photosphere. Another scientific objective is the plasma heating and acceleration processes and thermal inputs of flares and microflares and their fine magnetic field structures. Heating, acceleration, flares and microflares but also internal structure ( $g$-modes) are "big" questions that indeed, after years of limited observations, now deserve a dedicated and efficient program: ultra-high resolution imaging and spectro-polarimetry.

\subsection{High-resolution paradigms}

The supergranulation network, which dominates the chromospheric plasma dynamics, is apparent in the EUV emission pattern as seen by the SUMER instrument onboard SOHO shown in Fig. 2. Magnetograms from SOHO have revealed the ubiquitous appearance of small magnetic bipoles at the solar surface. After emergence, the polarities separate and are carried to the network boundaries by the supergranular flow, where they merge with the pre-existing network flux. This leads to flux cancellation, sub-mergence, and reconnection events. The magnetograms also show that 
the magnetic field exists in the network in two components side-by-side, i.e. in uncancelled unipolar fields or in a carpet of closed loops and flux tubes. The small loops will of course emerge or contract downwards and collide, and thus constitute a permanent source of energy, which can be tapped by the particles through magnetic field dissipation. Recent numerical simulations suggest that many of the bipolar structures can only be resolved at a resolution of $20 \mathrm{~km}$ or less [see e.g. 25, 31, 32, 33]

As a consequence of these observations, theoretical ideas about the origin of the solar wind have been put forward (see review of $[5,10,46]$ ) according to which the wind originates in the chromospheric network, and draws its energy from highfrequency waves generated by magnetic reconnections of the dynamic and complex fields prevailing there. Above mid-chromospheric altitudes the field expands rapidly, fills the overlying corona and guides the solar wind mass flux, emanating from the open chromosphere, where the plasma is created by photoionization. Plasma outflow has indeed been detected by SUMER on-board SOHO and is illustrated in Fig. 2. The mechanisms responsible for the origin of the slow solar wind remains unidentified. Small-scale reconnection, high altitude reconnection in streamers, spiculeinduced shocks, and magnetohydrodynamic (MHD) wave dissipation have been proposed [7, 33]. Different signatures are expected from each mechanism: small-scale jets, strong downflows in streamer legs, formation of shocks, or propagating waves, respectively. HiRISE with its proposed unprecedented spatial resolution will for the first time: a) reveal the fine structure of the network and provide definitive answers to the question of where and how the solar wind originates; b) allow images and 2D spectro-imaging to reliably disentangle spatial and temporal structures; c) observe the source signatures of the plasma (reconnection, dissipation). EUV spectral lines will play a key role with temperature and velocities diagnostics.

For example, small-scale magnetic activity is expected to continually produce waves, energetic particles, and rapidly-moving plasma. The dissipation of the waves could involve cyclotron damping. This process is observed to operate in the distant solar wind [58] and known to heat the plasma. Detailed observations of such key plasma processes originating in the transition region and heating the extended outer corona afterwards are now needed.

The magnetohydrodynamic waves generated in the photosphere by convective motions of the granules and supergranules are primarily of low frequency. In the small-scale magnetic structures of the strongly inhomogeneous network fields higher-frequency waves could be excited up to the $\mathrm{kHz}$ range. Such waves would certainly transfer very effectively wave energy, e.g. into the transverse kinetic degrees of freedom of the protons, and particularly the heavy ions, thereby heating them to very high coronal temperatures, a process for which the UVCS instrument and the SUMER experiment on SOHO, or lately Hinode, have found evidence in the strong Doppler-broadenings of emission lines [3, 7, 35, 63, 66]. Due to its investigation potential in the UV, transition zone heating sites of the corona, and the high sensitivity and spatial resolution of its instrumentation, HiRISE will for the first time be able to see very dim emissions, concentrated for high contrast in emissivity, for instance in plasma confined in small loops (perhaps in threads of 10-20 km), or loop profiles [53]. 

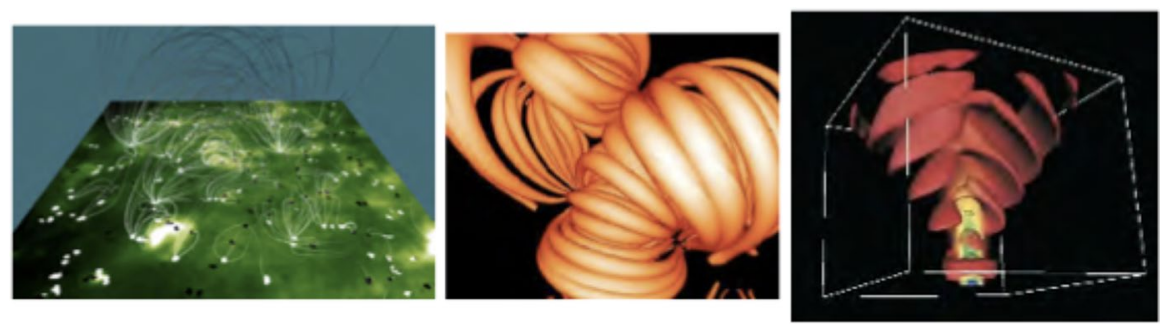

Fig. 3 Modeling magnetic fields extension in the chromosphere and corona is now possible and will gain from higher resolution and atmospheric height sampling. In order to extrapolate with confidence to the corona, vector magnetic fields are required in the photosphere (left, courtesy of A. Title, LMSAL, USA); direct magnetic field measurement in the corona would help to validate the extrapolations (see the simulations middle/right)

The major part of the magnetic flux permeating the solar photosphere outside sunspots is concentrated in small (scales of $20 \mathrm{~km}$ or so) flux tubes of kilo-gauss field strength. The structure and dynamics of these fundamental elements of the near-surface magnetic field has profound implications for a number of basic questions, e.g.:

- how do magnetic foot-point motion, wave excitation, flux cancellation, and reconnection contribute to the flux of mechanical energy into the corona?

- in which way do the emergence, evolution, and removal of magnetic flux elements determine the magnetic flux budget of the Sun? Is there a local dynamo operating on the scale of granulation?

- what is the origin of the facular contribution to the variability of the Solar constant?

- what is the physics of the interaction between convection and magnetism?

Answers to the questions require the study of magnetic flux elements on their intrinsic spatial scale. The high-resolution of the imagers, spectro-imagers, and magnetograph is intended to monitor the emergence, dynamics, twist, shearing, mutual interactions, and possible coalescence and subduction below the surface in order to follow the evolution and scrutinize the life cycles of magnetic flux elements. Coupled with the resolved oscillations of HiRISE instruments like DMOF (a Dual Magneto Optical Filter), the rotation below the surface can be addressed and, accordingly, the relation between convection and magnetism. Tangled magnetic fields associated with MHD waves and electric currents are believed to play a dominant role in the magnetic field restructuring, leading to energy dissipation. Modeling (see examples in Fig. 3) has significantly progressed over the last years and delivered promising "paradigms" for this dissipation, either from tangled fields, as suggested and studied by $[33,34]$, by leakage of oscillations (waves) at the level of the upper chromosphere and transition zone (and in spicules) as studied by e.g. [19-21, 23-25, 31], or even by direct convection simulations like in the work of [49-51]. These approaches to energy dissipation and the creation of fine structures (observed in the 
transition zone by, e.g., [21-23], have produced some evidence but desperately need to be disentangled by direct observations with higher spectral and spatial resolution and direct measurements of the magnetic field.

A key scientific objective of HiRISE is to study the new emergence and the cancellation of photospheric magnetic flux (the latter is the disappearance of opposite polarity regions in close contact), and to investigate the consequences of such processes for the overlying global coronal magnetic loops and for the chromospheric and transition region magnetic network above. Flux cancellations are known to be associated with or at the origin of various active phenomena, such as filament formation and eruption, evolution of small points of emission bright in radio or X-rays, or the occurrence of flares. Magnetograms combined with UV, FUV, EUV, and soft $\mathrm{X}$-ray images as well as FUV and EUV spectra, and the direct magnetic field measurements in the inner corona by e.g. a Lyman coronagraph channel, are the key data necessary to understand the bearing that small scale magnetic activity has on the transition zone and inner corona.

The Yohkoh, SOHO, TRACE, Hinode, and now SDO extreme ultraviolet and soft $\mathrm{X}$-ray telescopes have provided and still provide a rich harvest of coronal images, though only at a spatial resolution not much better than 1 arcsec. These images (see, for example, $[57,59])$ illustrate the existence of fine-scale structures in the corona, such as polar plumes and thin post-flare loops, and reveal continuous dynamics occurring on all resolved scales in particular the finest. There is also strong evidence that the actual brightness structures lie well below the best current spatial resolution. This points to the need for still higher spatial resolution. The active Sun has still not been imaged with sufficient resolution to address many of the important open questions, some listed above. The early triggering phase of solar flares and small activity regions and the evolution of point-like events or bright X-ray spots will be monitored, with simultaneous observations in relevant wavelengths and foot-point (photospheric and chromospheric) magnetic field measurements being carried out (see also [1]). The wide coverage of coronal temperatures by the visible-light, UV, FUV, EUV, and X-ray telescopes would enable complete images to be obtained in fast cadence. From these, the density and temperature distributions can reliably be derived, such that the traits of coronal heating processes in current sheets, shock fronts, or acceleration in small explosive events and rapid plasma jets might become clearly visible and be resolved in time and space.

\subsection{Coronal dynamics, heating, and magnetic field}

The energy that heats the corona and accelerates the solar wind and coronal mass ejections (CMEs) originates in sub-photospheric convective motions. The physical processes that transport this energy to the corona and convert it into thermal, kinetic, and magnetic energy are not fully understood. Space missions, and in particular SOHO and Hinode, have greatly advanced our knowledge about coronal heating, solar wind acceleration, and CMEs, but many key questions remain unanswered. A detailed understanding of physical processes in the corona is important not only for explaining the origins of space weather, but also for establishing a baseline of 
knowledge in plasma physics that is directly relevant to the Sun, other stars, and astrophysical systems ranging from the interstellar medium to black hole accretion discs.

Different physical mechanisms for heating the corona probably govern closed magnetic loops, active regions, and the open field lines that give rise to the solar wind $[54,60]$. Both densities and volumetric heating rates decrease rapidly with distance from the photosphere, but the coronal heating rates per particle remain large in the extended corona $[10,68]$. This, combined with remote and in situ evidence that heating continues in interplanetary space [42], implies that processes responsible for heating, wind acceleration, and CME ejection are dynamically important below and above the coronal base. An empirical description of the primary acceleration region (in the transition region and inner corona) of the wind and CMEs is especially crucial to determining how plasma properties at 1 AU are established.

We now need to test ion-cyclotron heating in coronal holes: the width of the O VI line in SOHO/UVCS observations increased dramatically in the range 1.5-2 solar radii $\left(R_{S}\right)$ above the polar coronal holes. This line width has been interpreted as the signature of the dissipation of high-frequency collisionless ion-cyclotron waves. In particular, the EUDS spectrometer on HiRISE will characterise the line width of O VI, Mg X and other emission lines (e.g. S VI, Fe XII, O V, He II, S X, Ne VIII, Si $\mathrm{XII})$ in polar coronal holes in the range 1.5-2 solar radii. Each of these ions is resonant with a different portion of the ion-cyclotron wave spectrum. Measurements of their line widths therefore provide a test of the ion-cyclotron wave theory. They will also test alternative interpretations that suggest the line width dependence on the adopted coronal electron density profiles [55].

There is a growing realization that the innermost 0.5 solar radii of the solar atmosphere is dominated by different physics than the extended corona at larger heliocentric distances. The chromosphere, transition region, and coronal base are strongly collisional and exhibit a complex magnetic topology, whereas the extended corona is relatively collisionless and uniform, with expansion of field lines into interplanetary space. Despite these local differences, the extended corona must be driven by energy that has made its way out through the lower layers. The lower solar atmosphere and corona are indeed efficiently coupled by the transport of non-thermal energy from the convection zone and by downward thermal conduction of heat from the corona. The nature of the outward energy flux is not known, but tangled magnetic fields associated with MHD waves and electric currents are believed to play a dominant role, as we saw previously [24, 25, 33, 34, 51, 61]. Dissipation of this energy, from tangled fields, foot-point convective motions, or waves - the three "theoretical paradigms" of heating to disentangle by direct observations - heats the coronal plasma along single or multi-threads [56], drives the solar wind, and propels CMEs. Understanding the physics of the characteristically different regions of the atmosphere necessitates studying these regions on structurally important scales, matching the 10-20 km spatial scale of magnetic elements in the solar photosphere. Studies of the temperature distribution of the corona indicate that the filling factor of emissive structures is $8 \%$ of the area resolved by EIS [67]. Resolving structures on a scale of 0.1 " to 0.7 " in the transition region and corona would allow us to probe these fundamental elements of the atmosphere's structure. Additionally, recent studies of 
flows in active regions $[9,48]$ indicate that there may be spatially unresolved flows near key magnetic domain interfaces [6] at which there are changes in the characteristic properties of the atmosphere such as temperature and density. Measuring these properties along with bulk velocity, through the transition region, where changes of regime are significant, is key to building working pictures of this interface, and thus to connect the high-beta and low-beta parts of the solar atmosphere.

The question of impulsive heating versus wave heating can also be approached with much more confidence at sub-arcsecond scales. In the lower chromosphere, the ability to spatially resolve coherent wave motions at sub-arcsecond scales has already been shown to be of great use in estimating energy flux in these relatively small-scale magnetic concentrations [32]. However, stable observing conditions and visibility across a greater height (and thus temperature) range in the solar atmosphere are required if we are to understand the characteristic energy flux in more than a handful of such elements. This requires moving to the FUV and EUV ranges. Spatial resolution and ability to measure dynamics and diagnostics in lines formed from the lower transition region (C III, H I) to the lower corona $(\mathrm{Mg} \mathrm{X})$ will allow us to measure the propagation of energy in waves through these regimes and measure the resulting energy absorbed by the local plasma. In this way, we can understand the contribution of these and other fine scale structures, such as those seen in active regions, to the energy flux that passes into the corona and solar wind.

Plasma flows are key signatures of non-thermal energy transport by wave propagation and of energy dissipation by magnetic reconnection. Observation of these flows in the transition region and corona is essential to address sources of coronal activity - heating, transient events, particle acceleration, solar wind, and irradiance variations. Through measuring line profiles and line intensities of several tens of EUV lines, these would provide answers to many long-standing and challenging questions.

While magnetic fields emerging in the solar atmosphere control the structure, dynamics, and heating of the solar corona, these fields remain essentially unattainable with the present low corona instrumentation. "Indeed, after 40 years of space coronagraphy, the lower corona $\left(<2.5 R_{S}\right)$ remains practically unobserved since LASCO-C1 on SOHO, aimed at observing the inner corona (with a Fabry-Perot setup), had a fairly large level of instrumental straylight, and since its Fabry-Perot did not survive the SOHO hibernation. The COR1 coronagraph on Secchi/STEREO are simple imaging systems with no diagnostic capability, and also suffer from large instrumental straylight. Ground-based coronagraphs are affected by seeing and atmospheric conditions, and are practically limited to a FOV $<1.5 R_{S}$. These observations are further background limited as Rayleigh scattering of the Earth atmosphere always dominates the flux received by the instrument. Full Stokes polarimetry of the Fe XIII $1074.7 \mathrm{~nm}$ line, giving information on the line of sight magnetic field magnitude, were obtained only recently [40, 41, 61] but are limited to bright, low altitude regions of the corona.

Performing high spatial resolution imaging of the corona as well as 2-dimensional spectroscopy of several emission lines from the transition region and corona base out to $2.5 R_{S}$ is necessary to address the following questions," [as quoted from page 8. from our own HiRISE Cosmic Vision proposal (2007)]: 
Fig. 4 Composite image of the corona of the August 11th, 1999 eclipse in Iran. The inner part is made of the White Light image obtained with a radial gradient neutral filter. The outer part is the LASCO-C2 (SOHO) image (adopted from [36]). Note the perfect correspondence of the radial fine structures. The proposed "SuperASPIICS" HiRISE coronagraph is intended to achieve the same quality as the eclipse observation in the inner corona with, in addition, unsurpassed diagnostic capability

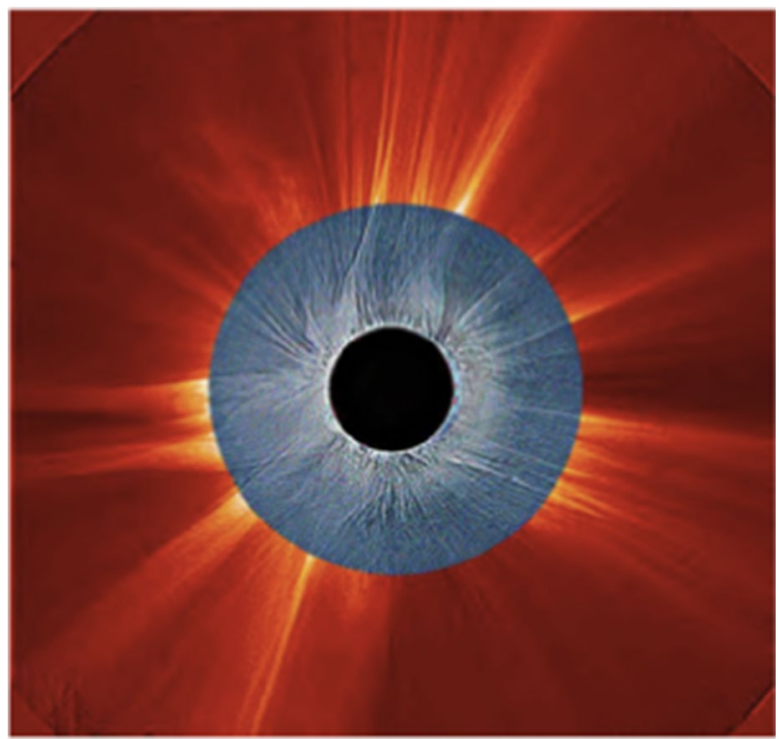

- How is the corona heated? What is the role of convective motions and of waves?

- How are the different components of the solar wind, slow and fast, accelerated?

- How are CMEs accelerated?

- What is the strength and direction of the coronal magnetic field? What is its topology?

To access the inner corona (see Fig. 4) uninterrupted with ultra-high spatio-temporal resolution, formation flying is an excellent solution, allowing visible spectroscopic measurements far out in the corona and near-infrared spectroscopic measurements in the low corona, and to perform magnetic field measurements. CMEs need to be observed to distinguish theories of CME initiation: the breakout model, the tether cutting model, and the flux rope model [47]. Observing evolution of the filament structure prior to eruption, recording high-speed jets that mark the reconnection site and observing the energy distribution in the post-eruptive plasma will provide discrimination between the models. We need to test theories of particle acceleration by observing pre-shock conditions and shock structure in the low corona with attempting to answer the question of how do shocks accelerate the corona. In particular, acceleration and injection mechanisms for impulsive Solar Energetic Particle (SEP) events are controversial. Wave-particle interactions or even CME-driven shocks have been proposed as the source of the enhancement. Measuring both X-rays and gamma rays from $10 \mathrm{keV}$ to an impressive $600 \mathrm{MeV}$ for large flaring events will confirm theories. Acceleration in shocks at heights $1.5-2 R_{S}$ will be characterised by measuring temperature increase of hotter lines ( $\mathrm{Mg} \mathrm{X}, \mathrm{Si}$ XII) or increase in line broadening (e.g. O VI). In the lower corona we need to remotely determine the elemental composition of plasma, in particular, of solar wind source regions to compare with 
in-situ measurements in the solar wind made by other spacecraft (Solar Probe Plus and/or InterHelioProbe).

\subsection{Solar interior and subsurface characterization}

The two major objectives in helioseismology are: first, to detect the gravity modes ( $g$-modes) of the Sun; second, to build upon MDI/SOHO and HMI/SDO with an improved resolved velocity imager at higher resolution (1 arcsec) and with improved magnetic field measurements, the latter to trace the magnetic field from the convective zone to the surface (local helioseismology; perhaps detecting flows from equator to the poles over the cycle).

$g$-modes are of prime importance to understand the structure and dynamics of the solar core which cannot be studied by using solar pressure modes ( $p$-modes) alone. So far the $g$-modes have not been discovered by any set of instruments on-board the SOHO spacecraft. After more than 10 years, GOLF has still only observed some weak possible signatures. The 1- $\sigma$ upper limit of $g$-mode amplitude at around 200 $\mu \mathrm{Hz}$ is typically $1 \mathrm{~mm} / \mathrm{s}$ or $0.1 \mathrm{ppm}$ [27]. Given a velocity amplitude of $1 \mathrm{~mm} / \mathrm{s} \mathrm{at}$ $200 \mu \mathrm{Hz}$, the displacement of the solar surface would be about $1.6 \mathrm{ppm}$ which is equivalent to a variation of solar radius of about $2 \mu \operatorname{arcsec}[37]$.

Measuring intensity fluctuations at the solar limb that perturb the equivalent solar radius signal, [4] reported to have detected $p$-modes using the limb data of the LOI instrument (part of the VIRGO instrument on SOHO). In some cases the amplification with respect to full-disc integrated data is about 4 , i.e. it means that a $p$-mode with amplitude of $1 \mathrm{ppm}$ in full disc is observed with amplitude of $4 \mathrm{ppm}$ at the limb (cf. [17]). This amplification factor was predicted by theory [4]. A pessimistic derivation gave 20 years for the detection of the first few $g$-modes with SOHO ([27]). We confidently envisage detecting them in 16 months with the amplification factor provided by a high-resolution instrumentation.

Fundamental aspects. It is anticipated that the solar variability is driven by the Sun's internal dynamics that generates and interacts with the magnetic field, maintained by dynamo action. However, it is not easy to simulate those dynamics due to yet fairly poor observational constraints on parameters on the solar radiative zone, although of prime importance since this represents $98 \%$ of the solar mass $(71 \%$ in radius) Precise observations from space are probably our only chance to obtain direct and indirect information on the solar internal dynamics and magnetic fields down to the solar core.

The magnetic field is only directly constrained just below the photosphere. SDO improved our knowledge of the convective zone but has not addressed the radiative zone with needed accuracy in detecting the gravity modes that diagnose the radiative zone and the inner core (see Fig. 5, and [43]).

Magneto-helioseismology of the photosphere-chromosphere interface. The acoustic modes are very sensitive to the subsurface layers but global helioseismology has not extracted any information above $0.97 R_{S}$. This region benefits from the development of local helioseismology. One needs to better describe these layers and quantify the emergent magnetic field to understand the evolution of the total 

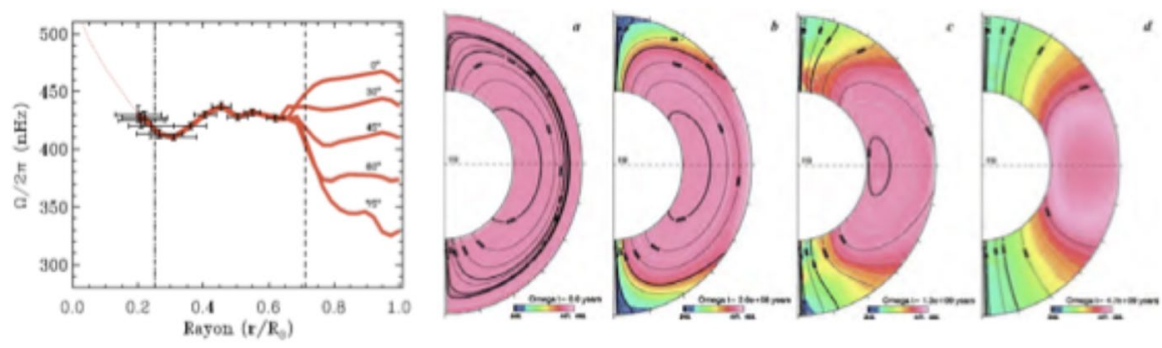

Fig. 5 Left: Solar rotation profile deduced from acoustic modes (MDI and GOLF, SOHO). Information is lacking and uncertain when reaching the core, leaving open all possible extrapolations. Right: a)-d) 3D MHD simulation of the impact of a fossil field located in the radiative zone, on the internal rotation profile. A better knowledge of the differential rotation in latitude and radius will largely constrain the 3D simulation and will help to answer the question: is there a dynamo effect in the radiative zone?

luminosity and of the radius from one solar cycle to another one or even along one solar cycle. Recent progress came from the surface gravity $f$-modes that represent the best tool to extract information from the sub-surface region. The temporal study of these modes over the solar cycle has revealed the presence of a double sheet: the region just below $0.99 R_{S}$ evolves in phase with the solar cycle, although the region above seems to be in phase opposition. A latitudinal study of these layers is extremely useful, and an independent measurement of the photospheric radius variation along the solar cycle useful to disentangle the different actors. Moreover, real constraints on the thermodynamics and magnetic properties of the region between the photosphere and the chromosphere will be possible through the analysis of several absorption lines: $\mathrm{He}, \mathrm{Na}, \mathrm{K}, \mathrm{Ca}$. Measurements in the $\mathrm{K}$ and $\mathrm{Na}$ lines would address these issues and waves propagation. One will see how the magnetic field modifies the density and pressure profile in the atmosphere, and we hope to identify and follow the chromospheric modes in this way.

\subsection{Variability, space weather, and space climate}

The irradiance of the Sun (i.e. its brightness as measured above the Earth's atmosphere) is known to vary by $0.1 \%$ over the solar cycle [26]. There is evidence for the UV component, which represents less than $10 \%$ of the total solar irradiance, to have a minor impact on regional climate. A crucial issue is the continuation of spectrallyresolved measurements of solar irradiance by several missions to allow for cross-calibration. Basic questions need to be answered before we can reach an understanding of the causes of solar irradiance variability:

- How does the solar luminosity (i.e. the radiation escaping in all directions) vary?

- Does it change at all or is a brightening at the equator compensated by a darkening over the poles?

- Why is the irradiance variability of the Sun a factor of three smaller than that of Sun-like stars? 
Since the solar energy is one of the major driving inputs for terrestrial climate it is important to know on what timescales the solar irradiance and other fundamental solar parameters, like the solar shape, vary in order to better understand and assess the origin and mechanisms of natural climate forcing.

Direct total solar irradiance measurements have been performed in space since 1978 by using radiometers. Reconstructions prior to the space age are today routinely performed by using either solar surface magnetism or by relying on combinations of solar proxies. The most commonly used proxies are the sunspot number and the facular coverage, which allow us to trace back to 1610 [39]. Combining these with cosmogenic radionuclide proxy records allows the total solar irradiance to be reconstructed over several millenia [71]. One of the main challenges is the quantification of the solar radiative output during periods of low solar activity, such as the Maunder minimum.

While the diameter does not vary significantly over the solar cycle whatever the technique used (fundamental mode, [2]: < 1 mas; MDI annulus images, [38]: < 7 mas; full MDI images, [13]: < 9 mas), neither over centuries by any significant amount ([62], who reanalysed Picard's data with the old instrument used by Picard himself: < 0.1" over 3 centuries; [69, 70], with "large" Gregorian telescopes nonaffected by seeing: $<0.05$ " over 30 years), it could be that the oblateness changes since we know that subsurface flows are active during the cycle (MDI results). These may or may not correlate with the cycle.

In order to establish without ambiguity the possible solar constant and solar shape (oblateness) relationship, a simultaneous measurement of both quantities from the same platform and in nonmagnetic lines or continua is required. The importance of the measurements for climatology is self-evident taking into account the extreme events like the Little Ice Age and the Maunder minimum.

UV and in particular Lyman- $\alpha$ irradiance has been monitored since 1977, and with the EOS/SOLSTICE experiment since 2002. Since these irradiance monitoring experiments observe the variability of the Sun as a star, there is no information about the physical causes of the observed spectral irradiance changes, notably in the UV $[44,45]$. To identify the causes of changes in Lyman- $\alpha$, but also the variability in the 200-220 nm continuum (Herzberg continuum, UV-ozone-climate interaction), one needs to compare the full disc irradiance data with images - both are required. High-resolution and continuous Lyman- $\alpha$ and Herzberg continuum images would enable progress to be made in these sciences. Such images would make it possible to better account for the observed Lyman- $\alpha$ changes and also for a better reconstruction of a long-term Lyman- $\alpha$ data set. Since Lyman- $\alpha$ irradiance is important for the ozone changes (as well as the Herzberg continuum) and the formation of the ionospheric D-region in the Earth's atmosphere, a better understanding of these variations will also be important for atmospheric science and aeronomy.

FUV/EUV observations are also likely to contribute to an important connection, namely that of the solar wind to space weather. A combination of recent results has also thrown light on the question of the behaviour of the solar wind. Studies of the magnetic topology of the periphery of solar active regions indicate that long field lines are anchored in these areas, perhaps extending to interplanetary space. Observations of hot, persistently upflowing material in these locations [29] suggest 
a source for the slow solar wind which is compounded by further remote-sensing results indicating that the composition of these upflows is consistent with that measured in-situ in the slow solar wind near the Earth [8]. Probing these regions at transition region temperatures will allow us to study in detail the locations where this material is released and to establish the veracity of such a tantalizing connection, not least its contribution to the variability of the slow solar wind and, thus, space weather in the terrestrial environment.

\section{Scientific requirements}

We have, in Section 2, outlined different needs and complementaries to achieve the global understanding of the Sun that we foresee, from the deep interior to the corona and heliosphere. A feasible payload (see Section 4.1) for the HiRISE mission concept is to have two satellites with 14 instruments together to address these topics with the ambitious and most appropriate and performing responses.

The need for very high spatial and spectral resolution at the chromosphere-corona interface is addressed by the $1.4 \mathrm{~m}$ interferometer SOLARNET and its spectroimaging instrument in the UV (UVIS). The need was outlined in the European ASTRONET $^{1}$ exercise which recommended a "meter class" FUV telescope. This scientific need is addressed in our mission concept by the UV interferometer of 1.4 meter baseline, and made of three "small" $500 \mathrm{~mm}$ diameter telescopes, perfectly phased, so as to act as a single, monolithic, telescope (compact configuration). These proposed interferometer technologies have been breadboarded by LATMOS and validated through an end-to-end test from object to image reconstruction, both in laboratory conditions, and directly observing the Sun. These achievements fully secure the development of the SOLARNET interferometer as both detailed and system design issues have been addressed also. A working three-telescope imaging interferometer is currently in use at LATMOS. This three-telescope interferometer, although "small" by ground standards, is large in terms of space hardware and in particular in solar physics since the largest telescope flown up to now is the $50 \mathrm{~cm}$ Hinode telescope. HiRISE ambitions are, however, timely since the SOLAR-C mission is also proposing a $1.5 \mathrm{~m}$ telescope. HiRISE has the advantage that the three telescopes can either work together or, independently and simultaneously, to optimize the observing capabilities. In the present mission concept we suggest the threetelescope interferometer is under ESA responsibility since it is too large for laboratories to realize, integrate, and test. In our view, only ESA and an industrial prime like Astrium or Thales Alenia Space can manage the task (and can handle the cost in view of the National Agencies present limitations).

Magnetic field direct measurement at photospheric level is covered on HiRISE with extreme precision by the 8-channel Vector Magnetograph (VM), completed by the DMOF in the low chromosphere and the UV channel of the giant, externally occulted, coronagraph (LCI of SuperASPIICS or CUSP) in the corona.

\footnotetext{
$\overline{1}$ https://www.astronet-eu.org/science-vision-infrastructure-roadmap-2020-2030
} 
CMEs and flares have imagers (SUAVE and the Heliospheric Large FOV Imager, HLI) and the High Energy Burst Spectrometers (HEBS) with unprecedented coverage of hard X-rays and gamma rays from $10 \mathrm{keV}$ to $600 \mathrm{MeV}$.

Solar variability, total and spectral, but also in other possible parameters, like the solar shape, are covered by PREMOS II, SWIP and SUAVE instruments.

Solar eclipses were, up to now, rare opportunities to access the inner corona since the scattering depends directly on the distance between the observer and the occulter. By using formation flying between two satellites $375 \mathrm{~m}$ apart, (occulting disc of $3.5 \mathrm{~m}$ on the main satellite), a giant externally occulted coronagraph is built, accessing the very near-limb of the Sun at 1.01 solar radii and with high resolution. Unique measurements are possible from the visible Fe XIV line, IR Fe XIII, and UV Lyman series and O VI.

Finally, the oscillation instruments allow to investigate, on one part, and with unprecedented performances, the deep interior for $g$-modes search with SUAVE (FUV imaging - variability and climate - and possible limb oscillations in the FUV), and the convective zone for local helioseismology with the Dual Magneto Optical Filter (DMOF). These instruments have been detailed already (see [14, 18], for SUAVE for example). In our view, cost constraints on National Agencies may however preclude their realisation if a bartering is to be done between extreme coronagraphy and oscillations.

To ease Sun-centered instrument mounting (helioseismology, variability) these small instruments could be mounted on a Hexapod pointing platform. Again, this is an expertise of ESA (the Hexapod platform for Sage III on the Space Station was provided by ESA to NASA) and the required signal to guide the Hexapod can directly come from the pointing telescope of SUAVE (similar to the guiding telescope of SODISM on the CNES PICARD mission ${ }^{2}$ that is driving the microsatellite platform).

A mission lifetime of three years could be realistically anticipated in the first instance, but with hardware and consumables sized for a 6-12 years mission, consistent with a solar maximum mission observing the solar cycle 25 , and an extended mission observing its decline.

The briefly outlined HiRISE concept could provide unique possibilities to do optical remote sensing observations of the Sun with unprecedented spatial resolution at scales below $20 \mathrm{~km}$ in images obtained in various wavelength bands. With the recent Hinode, SDO, and IRIS revelations of fine structures existing in the corona it is obvious that coronal imaging is crucial to put the filamentary structures observed in the solar wind, coronal loops, and inner heliosphere in their proper solar context.

An integrated ensemble of optical instruments would be envisioned which may be combined in several packages developed by large teams of scientists. Emphasis is put here on the scientific requirements and the presentation of feasible and proven designs to achieve them. All the imagers, beside the very high resolution of the interferometer, aim at high spatial $(0.5 \mathrm{arcsec})$ and temporal $(<1 \mathrm{~s})$ resolution, to reveal the context of the small-scale dynamical processes in the atmosphere and

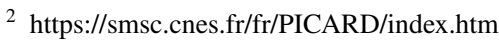


to study the rapid changes in morphology associated with transition region and coronal magnetic activity. Critical issues like heat ingress into the telescopes, aperture locations, and mechanical structures of the instruments, would be evaluated as part of a future feasibility study, and proven solutions would be implemented, for example, as in the CNES PICARD microsatellite programme (for example, using CarbonCarbon structure).

\section{Potential scientific model payload instruments}

\subsection{Scientific payload concept}

The dynamics of the chromosphere and corona are controlled by the emerging ubiquitous magnetic field. The plasma dynamics in each magnetic thread is believed to be linked to the formation of filaments, each one being dynamic on its own, in a non-equilibrium state. Mechanisms sustaining these dynamics, their manifestation in oscillations or waves (Alfvén or magneto-acoustic), require both very high-cadence, multi-spectral observations, and high resolution. Combining ultra-high resolution and coronagraphy one could achieve:

- resolution (0.02"), chromosphere-corona interface characterization with a $1.4 \mathrm{~m}$ UV-FUV telescope equivalent (three x $500 \mathrm{~mm}$ telescopes, independent or combined) and 3D imaging spectro-polarimetry;

- eclipse-like extreme coronagraphy of inner corona (up to $1.01 R_{S}$ ) in visible, UV, NIR by formation flying between two satellites $375 \mathrm{~m}$ apart: Fe XIV, He I D3 and 1083, Fe XIII 1075 and 79, Lyman series and O VI;

- continuity, waves and oscillations, global and local magnetoseismology, EUV and XUV complementary imagers and spectrometers, total solar irradiance (TSI) and photometers, solar spectral irradiance, and particles and magnetic fields experiments.

The HiRISE payload may be distributed on two satellites $375 \mathrm{~m}$ apart with respective payloads (instruments) of 500 and $150 \mathrm{~kg}$. The major payload includes the three telescopes of SOLARNET and ten instruments (three in the I2P, the Interferometer Instrument Package), in a volume of diameter $2.8 \mathrm{~m}$ by $3 \mathrm{~m}$ high:

SOLARNET Interferometer (three x $50 \mathrm{~cm}$ telescopes, $1.4 \mathrm{~m}$ baseline, actively

cophased and pointed)

$100 \mathrm{~kg}$

I2P: UV Imaging Spectrometer (UVIS) - subtractive double monochromator

and IFTS

$60 \mathrm{~kg}$

I2P: high resolution Vector Magnetograph (VM)

$60 \mathrm{~kg}$

I2P: Chromospheric Imaging Camera (CIC)

$40 \mathrm{~kg}$

EUV Diagnostic Spectrometer (EUDS)

$55 \mathrm{~kg}$

EUV and X-ray Imagers (EUXI)

$63 \mathrm{~kg}$ 
High Energy Burst Spectrometers (HEBS) hard X-rays and gamma rays, $10 \mathrm{keV}$

to $600 \mathrm{MeV}$

$20 \mathrm{~kg}$

Dual Magneto Optical Filter (DMOF) for full Sun magnetograph and oscillations $\quad 60 \mathrm{~kg}$

SUAVE, solar activity, limb oscillations, and shape changes

(full Sun Lyman Alpha, 160 and $220 \mathrm{~nm}$ imaging)

$25 \mathrm{~kg}$

SOLSIM, solar ultraviolet spectral irradiance monitor

$9 \mathrm{~kg}$

PREMOS II (TSI and Photometers with fast sampling rates and redundancy)

$8 \mathrm{~kg}$

On the Chaser satellite, in formation flying at $375 \mathrm{~m}$ (on a Herschel-like platform), we have:

$\begin{array}{ll}\text { SuperASPIICS Green, IR (1074.7 nm) and UV giant externally occulted } & \\ \text { coronagraph } & 120 \mathrm{~kg} \\ \begin{array}{l}\text { HLI, Heliospheric Large FOV }\left(20^{\circ}\right) \text { Imager } \\ \text { SWIP } \text { in-situ Solar Wind Instrument Package (magnetic field, shocks, thermal } \\ \text { plasma) }\end{array} & 20 \mathrm{~kg} \\ \end{array}$

The payload is a unique set of instruments to truly follow the magnetic field from its generation in the convective zone by local helioseismology, to its development and structuring in the chromosphere and transition zone, and energy release through waves, flux tubes motions, tangled magnetic field strands, or the combination of these processes in the corona. The very complete UV to near-IR externally occulted coronagraph allows imaging and spectroscopy (polarization) very near the limb in the inner corona. Direct magnetic field measurements are carried from the photosphere to the corona (Hanlé effect polarization of Lyman series).

\subsection{Payload technology developments}

Major technical advances are contained in the three-telescopes cophased and pointed system - an endeavour made possible by the success of the several ESA, CNES, and CNRS interferometry studies - and in the development of new instruments, which is expected since breakthrough instrumentation is a prerequisite for outstanding scientific outcome.

The important technologies needed by the proposed interferometer concept include stable structures and a cophasing system (phase measure and control). These technologies have been breadboarded by LATMOS with an end-to-end test from solar observation to image reconstruction. These achievements secure the development of the interferometer as both system design and detailed implementation issues are mastered. Among other interesting issues are progress made by the Turin group with Liquid-Crystal allowing to finely tune a narrow spectral range with polarization capabilities (SuperASPIICS instrument), and the new stable and long-duty (solar cycle) Lyman-Alpha telescope SUAVE now in a remarkably optimized off-axis configuration with SiC mirrors. 
Table 1 Potential mission summary table

\begin{tabular}{ll}
\hline Launch vehicle & $\begin{array}{l}\text { Soyuz-Fregat with } 2 \text { satellites in "stacked composite" configuration } \\
(<1700 \mathrm{~kg})\end{array}$ \\
Orbit & Halo orbit around the Lagrange L1 point \\
Formation Flying & two satellites $375 \mathrm{~m}$ apart controlled to 4-6 arcsec alignment \\
Spacecrafts & main satellite: Herschel platform re-use; \\
chaser satellite: Herschel-like platform (smaller: functional chains) & 3 years nominal (extended: $6-12$ years) - launch possible in 2029 \\
Mission duration & (solar cycle 25) \\
& $12 \mathrm{Mb} / \mathrm{s}$ downlink using Ka-band high rate modulation, \\
Data handling & a $40 \mathrm{~cm}$ antenna and the $35 \mathrm{~m}$ Cebreros antenna \\
& or New Norcia if upgraded at < 90\% capacity \\
\hline
\end{tabular}

\section{Potential mission configuration and profile}

\subsection{Potential mission profile summary}

HiRISE could achieve its impressive aims with a major mission scenario involving two satellites formation flying $375 \mathrm{~m}$ apart at the L1 Lagrangian point. The main spacecraft uses a modified Herschel platform, and the chaser spacecraft, forming a giant externally occulted coronagraph, uses a Herschel-like platform. A novelty would also be in state-of-the-art new instruments, allowing kilometric resolution and reasonable mass and volume, like the SOLARNET interferometer. The numerous high technologies, new features of HiRISE, would lead to novel insights into how the Sun works from the interior to the outer corona and to the Earth. With orders of magnitude improvement in spatial resolution but also in sensitivity (UV spectroimaging, diameter oscillations, and more), HiRISE is seen to have the potential to resolve major open science questions in modern solar physics and potentially lead to a wide variety of important discoveries. For the first time an ultra-high resolution formation flying facility would address directly the key component in understanding the Sun: the magnetic field structuring. It is anticipated that one would resolve it in much smaller features to analyze thoroughly the Sun's magnetic activity on multiple scales: time variability, evolution, and fine-scale structure of the dynamic chromosphere, transition region, and corona; origin, confinement, acceleration, and release of energy; and heating of the chromosphere and corona. HiRISE would also have complemented the in-situ observations of the Parker Solar Probe and fly-by of Solar Orbiter and build upon Hinode, SDO, and IRIS moderate spatial resolution observations (Table 1). 


\subsection{Mission and technology spacecraft challenges - science perspectives}

The main technologies necessary for the formation flying of the HiRISE concept will be mature at the time of phase A kick-off thanks to PRISMA ${ }^{3}$ and ASPIICS/ PROBA-3 in-orbit demonstrations [28].

- In the frame of the PRISMA mission, Thales Alenia Space has already fully demonstrated the RF metrology and the associated formation control modes and software.

- The HiRISE formation flying needs have to be fully covered by an appropriate technology development plan, including but not exclusively the following R\&D: i) RF Metrology; ii) Optical Longitudinal and Lateral metrology; iii) $\mu$-propulsion; iv) Formation flying avionics software; v) Ka band high rate modulator.

The formation flying technologies are the principal technology developments required for the mission; they are already funded through either national, ESA TRP or GSTP actions, ensuring their availability for the HiRISE mission.

The "Stacked Composite" approach, proposed and studied by Thales Alenia Space, for a launch by Soyuz to L1 of the two HiRISE satellites, is well dimensioned, and provides a comfortable margin (> 20\%). Telemetry is evaluated to 12 Mbits/s, which is indeed high but in the scope, for instance, of the Cebreros antenna Ka-band high rate modulator $41 \mathrm{Mbits} / \mathrm{s}$ (i.e. 13.6 Mbits/s on 8h/day). The expected volume and format of the data coming from the various instruments will be larger than any previous solar physics spacecraft but small compared to current Earth observation missions. No particular difficulties are foreseen to handle, process and archive the data.

The cost conscious approach of re-using Herschel and Herschel-like platforms allows a mission at the Lagrange point L1 with extreme coronagraphy thanks to formation flying. Costs have been thoroughly evaluated by Thales Alenia Space in 2007 (HiRISE Cosmic Vision proposal: 340 MEur) and revised since to 500 MEur taking into account M3 and M4 missions' evaluation by ESA in 2010 and 2015. This very reasonable mission cost results from the consequent heritage of the mission (Herschel platform in particular).

There is no scientific revolution without a technological one. Both formation flying - allowing ambitious coronagraphy near the limb - and interferometry on extended objects in the Far UV - allowing the energy release at the chromospherecorona interface to be probed - are building on this. The risk taken, which in our opinion is reasonable in respect of the precursors and technological developments, is worth the unique results anticipated, that none of the current or planned missions will bring. Though, we are conscious that the gain in high-resolution comes in part from the FUV access of our spectro-imaging approach and from the interferometer that makes the gain in resolution an estimated factor 3, i.e. from 60 to $20 \mathrm{~km}$, a major step to disentangle coronal heating theories but, also, a major cost and risk

\footnotetext{
${ }^{3}$ http://prisma-i.it/index.php/en/
} 
issue. Accordingly, and to minimize risk, the payload is using interferometry ultrahigh resolution as an extra. The three $x 50 \mathrm{~cm}$ telescopes can, indeed, be combined together towards the 2D UVIS spectrograph but they can also be used independently and simultaneously for multi-spectral multi-temperature sensing by the three instruments in the I2P since, in each telescope, light is directly linked to one major instrument: the FUV 2D spectrograph (UVIS), the Vector Magnetic Field Instrument (VM), and the Chromospheric Imaging Camera (CIC). This way, risk is minimized and innovation preserved with access to interferometry, a major key to discoveries. Nevertheless, a monolithic telescope option will also be considered as a trade-off even if heat evacuation is much more critical for larger telescopes.

\section{International collaborations}

HiRISE would serve a large community of more than 70 Co-Is in 15 countries around the world. In addition, we would anticipate a very significant participation of non-EU institutes in this project (perhaps, even up to three major instruments being provided by Asian partners), the payload can be extremely ambitious gaining orders of magnitude on previous recent missions (Hinode, STEREO, SDO, Parker Solar Probe, or Solar Orbiter).

Russian colleagues from the Lebedev Institute would also contribute a major instrument. The remaining cost for European countries intending to provide payloads would therefore be limited and reasonable ( $<200$ MEuro).

HiRISE at the L1 Lagrange point would also be highly complementary to the Solar Orbiter and Parker Solar Probe missions to which it brings coronagraphy, coronal magnetometry, and very high resolution in the upper chromosphere, transition zone, and inner corona where most of the coronal heating physics is thought to happen through flux tubes convective motions, waves, or magnetic reconnection of tangled magnetic field strands. HiRISE would build upon our European competencies in EUV/XUV imaging and spectroscopy, would enhance global and local helioseismology measurements, use the revolution in high resolution made possible by interferometry, and add the breakthrough of formation flying to achieve close limb coronagraphic observations. Furthermore, continuity of observation at the L1 Lagrange point could allow unprecedented new limb and velocity oscillations search for $g$-modes, to understand the magnetic origin of the solar activity leading, hopefully, to predictions of space weather for the coming decades.

\section{Conclusions}

In this White Paper, submitted in response to the ESA Voyage 2050 Call, we present our mission concept to measure a wide range of key solar parameters (magnetic and velocity fields, particle energy spectrum, total and spectral solar irradiance, etc.) to determine what drives the local and global dynamics from the photosphere interconnecting to the outer solar atmosphere. The full science return can only be achieved by an L-class mission given the key element of the proposed unique 
formation flying. With a downgrade to an M-class mission, other scenarios with a narrower scope might become possible. One downgrade option that could be instigated would be, in the context of Voyage2050, to combine our ultra-high resolution suggestion (downgraded to M-class) with one or more missions in the fields of solar, heliospheric, magnetospheric, and ionospheric physics as described in other White Papers submitted to the Voyage 2050 Call. ${ }^{4}$ This would provide a Grand European Heliospheric Observatory that not only addresses major challenges in the Solar-Terrestrial physics discipline but provides rapid scientific advances in a holistic approach to science that underpins our European space weather requirements for decades to come.

Acknowledgements The authors would like to thank the anonymous referee for the very careful reading of the paper and for suggesting structural changes for a more fluent read. Sources of funding acknowledged are: R.E. acknowledges the support received from the Science and Technology Facilities Council (STFC) UK (grant number ST/M000826/1 at the University of Sheffield). L.D. is grateful to the Centre National d'Etudes Spatiales (CNES) for R\&T support received to develop SOLARNET interferometric breadboard and new generation of FUV robust solar telescopes (SUAVE). M.B.K and HM are grateful to the Science and Technology Facilities Council (STFC), (UK, Aberystwyth University, grant number ST/ S000518/1). F.Z. acknowledges the support received by the Universitá degli Studi di Catania (Piano per la Ricerca Universitá di Catania 2016-2018 - Linea di intervento 2) and by the Italian MIUR-PRIN grant 2017APKP7T on Circumterrestrial Environment: Impact of Sun-Earth Interaction.

\section{Declarations}

Conflicts of interest Not applicable.

Open Access This article is licensed under a Creative Commons Attribution 4.0 International License, which permits use, sharing, adaptation, distribution and reproduction in any medium or format, as long as you give appropriate credit to the original author(s) and the source, provide a link to the Creative Commons licence, and indicate if changes were made. The images or other third party material in this article are included in the article's Creative Commons licence, unless indicated otherwise in a credit line to the material. If material is not included in the article's Creative Commons licence and your intended use is not permitted by statutory regulation or exceeds the permitted use, you will need to obtain permission directly from the copyright holder. To view a copy of this licence, visit http://creativecommons.org/licen ses/by/4.0/.

\section{References}

1. Amari, T., Canou, A., Aly, J.J.: Characterizing and predicting the magnetic environment leading to solar eruptions. Nature 514(7523), 465-469 (2014). https://doi.org/10.1038/nature13815

2. Antia, H.M.: Does the sun shrink with increasing magnetic activity? Astrophys. J. 590(1), 567-572 (2003). https://doi.org/10.1086/374916

3. Antonucci, E., Kohl, J.L., Noci, G., Tondello, G., Huber, M.C.E., Gardner, L.D., Nicolosi, P., Giordano, S., Spadaro, D., Ciaravella, A., Raymond, C.J., Naletto, G., Fineschi, S., Romoli, M., Siegmund, O.H.W., Benna, C., Michels, J., Modigliani, A., Panasyuk, A., Pernechele, C., Smith, P.L., Strachan, L., Ventura, R.: Velocity fields in the solar corona during mass ejections as observed with UVCS-SOHO. Astrophys. J. Lett. 490(2), L183-L186 (1997). https://doi.org/10.1086/311028

\footnotetext{
4 The White Papers submitted in response to the ESA Voyage 2050 call can be found at: https://www. cosmos.esa.int/web/voyage-2050/white-papers
} 
4. Appourchaux, T., Sekii, T., Gough, D., Lee, U., Wehrli, C., Virgo Team: Structure inversions with the VIRGO data. In: Provost, J., Schmider, F.X. (eds) Sounding Solar and Stellar Interiors, IAU Symposium, vol. 181, p. ISBN0792348389 (1997)

5. Axford, W.I., McKenzie, J.F.: The solar wind. In: Cosmic Winds and the Heliosphere, p. 31 (1997)

6. Baker, D., van Driel-Gesztelyi, L., Mandrini, C.H., Démoulin, P., Murray, M.J.: Magnetic reconnection along quasi-separatrix layers as a driver of ubiquitous active region outflows. Astrophys. J. 705(1), 926-935 (2009). https://doi.org/10.1088/0004-637X/705/1/926

7. Banerjee, D., Erdélyi, R., Oliver, R., O’Shea, E.: Present and future observing trends in atmospheric magnetoseismology. Solar Phys. 246(1), 3-29 (2007). https://doi.org/10.1007/s11207-007-9029-Z

8. Brooks, D.H., Warren, H.P.: Establishing a connection between active region outflows and the solar wind: abundance measurements with EIS/Hinode. Astrophys. J. Lett. 727(1), L13 (2011). https:// doi.org/10.1088/2041-8205/727/1/L13

9. Bryans, P., Young, P.R., Doschek, G.A.: Multiple component outflows in an active region observed with the EUV imaging spectrometer on hinode. Astrophys. J. 715(2), 1012-1020 (2010). https://doi. org/10.1088/0004-637X/715/2/1012

10. Cranmer, S.R.: Self-consistent models of the solar wind. Space Sci. Rev. 172(1-4), 145-156 (2012). https://doi.org/10.1007/s11214-010-9674-7

11. Damé, L.: A very high resolution vision for solar physics: Interferometry \& spectral-imaging in the far ultraviolet. In: SOHO-17. 10 Years of SOHO and Beyond, vol. 617, p. 35. ESA Special Publication (2006)

12. Damé, L.: Future solar space missions to observe the sun from the chromosphere to the corona and beyond. In: Damé, L., Hady, A. (eds.) International Association of Geomagnetism and Aeronomy (IAGA) 2nd Symposium: Solar Wind - Space Environment Interaction. Cairo University Press (2010a) https://doi.org/10.1012/S120027852002

13. Damé, L.: The solar diameter is most probably constant over the solar cycle. In: 38th COSPAR Scientific Assembly, vol. 38, p. 2 (2010b)

14. Damé, L.: The space weather and ultraviolet solar variability (SWUSV) microsatellite mission. Journal of Advanced Research 4(3), 235-251 (2013). https://doi.org/10.1016/j.jare.2013.03.002

15. Damé, L., Derrien, M.: Solar physics and interferometry mission (SPI). Advances in Space Research 29(12), 2061-2073 (2002). https://doi.org/10.1016/S0273-1177(02)00152-7

16. Damé, L., Derrien, M., Kozlowski, M., Merdjane, M.: High resolution solar physics by interferometry: SOLARNET. In: Crossroads for European Solar and Heliospheric Physics. Recent Achievements and Future Mission Possibilities, vol. 417, p. 109. ESA Special Publication (1998)

17. Damé, L., Hersé, M., Thuillier, G., Appourchaux, T., Crommelynck, D., Dewitte, S., Joukoff, A., Fröhlich, C., Laclare, F., Delmas, C., Boumier, P.: PICARD: simultaneous measurements of the solar diameter, differential rotation, solar constant and their variations. Advances in Space Research 24(2), 205-214 (1999). https://doi.org/10.1016/S0273-1177(99)00502-5

18. Damé, L., Meftah, M., Irbah, A., Hauchecorne, A., Keckhut, P., Quémerais, E.: SUAVE: a UV telescope for space weather and solar variability studies. In: Space Telescopes and Instrumentation 2014: Ultraviolet to Gamma Ray, Society of Photo-Optical Instrumentation Engineers (SPIE) Conference Series, vol. 9144, p. 914431 (2014) https://doi.org/10.1117/12.2057705

19. De Pontieu, B., Tarbell, T., Erdélyi, R.: Correlations on arcsecond scales between chromospheric and transition region emission in active regions. Astrophys. J. 590(1), 502-518 (2003). https://doi. org/10.1086/374928

20. De Pontieu, B., Erdélyi, R., James, S.P.: Solar chromospheric spicules from the leakage of photospheric oscillations and flows. Nature 430(6999), 536-539 (2004). https://doi.org/10.1038/natur e02749

21. De Pontieu, B., McIntosh, S.W., Carlsson, M., Hansteen, V.H., Tarbell, T.D., Schrijver, C.J., Title, A.M., Shine, R.A., Tsuneta, S., Katsukawa, Y., Ichimoto, K., Suematsu, Y., Shimizu, T., Nagata, S.: Chromospheric Alfvénic waves strong enough to power the solar wind. Science 318(5856), 1574 (2007). https://doi.org/10.1126/science.1151747

22. De Pontieu, B., Carlsson, M., Rouppe van der Voort, L.H.M., Rutten, R.J., Hansteen, V.H., Watanabe, H.: Ubiquitous torsional motions in Type II spicules. Astrophys. J. Lett. 752(1), L12 (2012). https://doi.org/10.1088/2041-8205/752/1/L12

23. De Pontieu, B., Title, A., Carlsson, M.: Probing the solar interface region. Science 346(6207), 315 (2014). https://doi.org/10.1126/science.346.6207.315

24. Erdélyim, R.: Magnetic seismology of the lower solar atmosphere. In: Proceedings of SOHO 18/ GONG 2006/HELAS I, Beyond the spherical Sun, vol. 624, p. 15. ESA Special Publication (2006) 
25. Erdélyi, R., Malins, C., Tóth, G., de Pontieu, B.: Leakage of photospheric acoustic waves into nonmagnetic solar atmosphere. Astron. Astrophys. 467(3), 1299-1311 (2007). https://doi.org/10.1051/ 0004-6361:20066857

26. Ermolli, I., Matthes, K., Dudok de Wit, T., Krivova, N.A., Tourpali, K., Weber, M., Unruh, Y.C., Gray, L., Langematz, U., Pilewskie, P., Rozanov, R., Schmutz, W., Shapiro, A., Solanki, S.K.: Recent variability of the solar spectral irradiance and its impact on climate modelling. Atmospheric Chemistry and Physics 12(8), 3945-3977 (2013). https://doi.org/10.5194/acp-13-3945-2013

27. Fröhlich, C., Finsterle, W., Andersen, B., Appourchaux, T., Chaplin, W.J., Elsworth, Y., Gough, D.O., Hoeksema, J.T., Isaak, G.R., Kosovichev, A.G., Provost, J., Scherrer, P.H., Sekii, T., Toutain, T.: Observational upper limits for low-degree solar $g$-modes. In: Korzennik, S. (ed.) Structure and Dynamics of the Interior of the Sun and Sun-like Stars, vol. 418, p. 67. ESA Special Publication (1998)

28. Galano, D., Bemporad, A., Buckley, S., Cernica, I., Dániel, V., Denis, F., de Vos, L., Fineschi, S., Galy, C., Graczyk, R., Horodyska, P., Jacob, J., Jansen, R., Kranitis, N., Kurowski, M., Ladno, M., Ledent, P., Loreggia, D., Melich, R., Mollet, D., Mosdorf, M., Paschalis, A., Peresty, R., Purica, M., Radzik, B., Rataj, M., Rougeot, R., Salvador, L., Thizy, C., Versluys, J., Walczak, T., Zarzycka, A., Zender, J., Zhukov, A.: Development of ASPIICS: a coronagraph based on Proba-3 formation flying mission. In: Lystrup, M., MacEwen, H.A., Fazio, G.G., Batalha, N., Siegler, N., Tong, E.C. (eds.) Space Telescopes and Instrumentation 2018: Optical, Infrared, and Millimeter Wave, Society of Photo-Optical Instrumentation Engineers (SPIE) Conference Series, vol. 10698, p. 106982 Y (2018) https://doi.org/10.1117/12.2312493

29. Harra, L.K., Sakao, T., Mandrini, C.H., Hara, H., Imada, S., Young, P.R., van Driel-Gesztelyi, L., Baker, D.: Outflows at the edges of active regions: contribution to solar wind formation? Astrophys. J. Lett. 676(2), L147 (2008). https://doi.org/10.1086/587485

30. Hassler, D.M., Dammasch, I.E., Lemaire, P., Brekke, P., Curdt, W., Mason, H.E., Vial, J.C., Wilhelm, K.: Solar wind outflow and the chromospheric magnetic network. Science 283, 810 (1999). https://doi.org/10.1126/science.283.5403.810

31. Jefferies, S.M., McIntosh, S.W., Armstrong, J.D., Bogdan, T.J., Cacciani, A.R., Fleck, B.: Magnetoacoustic portals and the basal heating of the solar chromosphere. Astrophys. J. Lett. 648(2), L151L155 (2006). https://doi.org/10.1086/508165

32. Jess, D.B., Mathioudakis, M., Erdélyi, R., Crockett, P.J., Keenan, F.P., Christian, D.J.: Alfvén waves in the lower solar atmosphere. Science 323(5921), 1582 (2009). https://doi.org/10.1126/science. 1168680

33. Klimchuk, J.A.: On solving the coronal heating problem. Solar Phys. 234(1), 41-77 (2006). https:// doi.org/10.1007/s11207-006-0055-Z

34. Klimchuk, J.A., López Fuentes, M.C.: Coronal heating. In: Solomos, N. (ed.) Recent Advances in Astronomy and Astrophysics, American Institute of Physics Conference Series, vol. 848, pp. 55-63 (2006) https://doi.org/10.1063/1.2347961

35. Kohl, J.L., Noci, G., Antonucci, E., Tondello, G., Huber, M.C.E., Gardner, L.D., Nicolosi, P., Strachan, L., Fineschi, S., Raymond, J.C., Romoli, M., Spadaro, D., Panasyuk, A., Siegmund, O.H.W., Benna, C., Ciaravella, A., Cranmer, S.R., Giordano, S., Karovska, M., Martin, R., Michels, J., Modigliani, A., Naletto, G., Pernechele, C., Poletto, G., Smith, P.L.: First Results from the SOHO Ultraviolet Coronagraph Spectrometer. Solar Phys. 175(2), 613-644 (1997). https://doi.org/10.1023/A: 1004903206467

36. Koutchmy, S., Baudin, F., Bocchialini, K., Daniel, J.Y., Delaboudiniére, J.P., Golub, L., Lamy, P., Adjabshirizadeh, A.: The August 11th, 1999 CME. Astron. Astrophys. 420, 709-718 (2004). https:// doi.org/10.1051/0004-6361:20040109

37. Kuhn, J.R., Bogart, R., Bush, R., Sá, L., Scherrer, P., Scheick, X.: Precision solar astrometry from SoHO/MDI. In: Provost, J., Schmider, F.X. (eds.) Sounding Solar and Stellar Interiors, IAU Symposium, vol. 181, p. ISBN0792348389 (1997)

38. Kuhn, J.R., Bush, R.I., Emilio, M., Scherrer, P.H.: On the constancy of the solar diameter. II. Astrophys. J. 613(2), 1241-1252 (2004). https://doi.org/10.1086/423301

39. Lean, J.: The Sun's variable radiation and its relevance for Earth. Ann. Rev. of Astron. Astrophys. 35, 33-67 (1997). https://doi.org/10.1146/annurev.astro.35.1.33

40. Lin, H., Penn, M.J., Tomczyk, S.: A new precise measurement of the coronal magnetic field strength. Astrophys. J. Lett. 541(2), L83-L86 (2000). https://doi.org/10.1086/312900

41. Lin, H., Kuhn, J.R., Coulter, R.: Coronal magnetic field measurements. Astrophys. J. Lett. 613(2), L177-L180 (2004). https://doi.org/10.1086/425217 
42. Marsch, E.: Cyclotron heating of the solar Corona. Astrophys. Space Sci. 264, 63-76 (1999). https:// doi.org/10.1023/A:1002436407996

43. Mathur, S., Turck-Chièze, S., Couvidat, S., García, R.A.: On the characteristics of the Solar gravity mode frequencies. Astrophys. J. 668(1), 594-602 (2007). https://doi.org/10.1086/521187

44. Meftah, M., Bolsée, D., Damé, L., Hauchecorne, A., Pereira, N., Irbah, A., Bekki, S., Cessateur, G., Foujols, T., Thiéblemont, R.: Solar irradiance from 165 to $400 \mathrm{~nm}$ in 2008 and UV variations in three spectral bands during solar cycle 24. Solar Phys. 291(12), 3527-3547 (2016). https://doi.org/ 10.1007/s11207-016-0997-8

45. Meftah, M., Damé, L., Bolsée, D., Hauchecorne, A., Pereira, N., Sluse, D., Cessateur, G., Irbah, A., Bureau, J., Weber, M., Bramstedt, K., Hilbig, T., Thiéblemont, R., Marchand, M., Lefèvre, F., Sarkissian, A., Bekki, S.: SOLAR-ISS: A new reference spectrum based on SOLAR/SOLSPEC observations. Astron. Astrophys. 611, A1 (2018). https://doi.org/10.1051/0004-6361/ 201731316

46. Ofman, L.: Wave modeling of the Solar wind. Living Reviews in Solar Physics 7(1), 4 (2010). https://doi.org/10.12942/lrsp-2010-4

47. Patsourakos, S., Vourlidas, A., Török, T., Kliem, B., Antiochos, S.K., Archontis, V., Aulanier, G., Cheng, X., Chintzoglou, G., Georgoulis, M.K., Green, L.M., Leake, J.E., Moore, R., Nindos, A., Syntelis, P., Yardley, S.L., Yurchyshyn, V., Zhang, J.: Decoding the pre-eruptive magnetic field configurations of coronal mass ejections. Space Sci. Rev. 216(8), 131 (2020). https://doi. org/10.1007/s11214-020-00757-9

48. Peter, H.: Asymmetries of solar coronal extreme ultraviolet emission lines. Astron. Astrophys. 521, A51 (2010). https://doi.org/10.1051/0004-6361/201014433

49. Peter, H., Gudiksen, B.V., Nordlund, A.: Coronal heating through braiding of magnetic field lines synthesized coronal EUV emission and magnetic structure. In: Innes, D.E., Lagg, A., Solanki, S.A. (eds.) Chromospheric and Coronal Magnetic Fields, vol. 596, p. 14.1. ESA Special Publication (2005a)

50. Peter, H., Gudiksen, B.V., Nordlund, Å.: EUV Emission from a 3D MHD coronal model: Temporal variability in a synthesized Corona. In: Fleck, B., Zurbuchen, T.H., Lacoste, H. (eds.) Solar Wind 11/SOHO 16, Connecting Sun and Heliosphere, vol. 592, p. 527 ESA Special Publication (2005b)

51. Peter, H., Gudiksen, B.V., Nordlund, Å.: Forward modeling of the Corona of the Sun and Solar-like stars: from a Three-dimensional magnetohydrodynamic model to synthetic extreme-ultraviolet spectra. Astrophys. J. 638(2), 1086-1100 (2006). https://doi.org/10.1086/499117

52. Peter, H, Abbo, L, Andretta, V, Auchère, F, Bemporad, A, Berrilli, F, Bommier, V, Braukhane, A, Casini, R, Curdt, W, Davila, J, Dittus, H, Fineschi, S, Fludra, A, Gandorfer, A, Griffin, D, Inhester, B, Lagg, A, Land i Degl'Innocenti, E, Maiwald, V, Sainz, RM, Martínez Pillet, V, Matthews, S, Moses, D, Parenti, S, Pietarila, A, Quantius, D, Raouafi, NE, Raymond, J, Rochus, P, Romberg, O, Schlotterer, M, Schühle, U, Solanki, S, Spadaro, D, Teriaca, L, Tomczyk, S, Trujillo Bueno, J, Vial, JC: Solar magnetism eXplorer (SolmeX). Exploring the magnetic field in the upper atmosphere of our closest star . Experimental Astronomy 33(2-3):271-303 (2012) https://doi.org/10.1007/ s10686-011-9271-0

53. Priest, E.R.: Heating the Solar Corona by magnetic reconnection. Astrophys. Space Sci. 264, 77-100 (1999). https://doi.org/10.1023/A:1002440524834

54. Priest, E.R.: How is the Solar Corona heated? In: Butler, C.J., Doyle, J.G. (eds.) Solar and stellar activity: similarities and differences, astronomical society of the pacific conference series, vol. 158 , p. 321 (1999b)

55. Raouafi, N.E., Solanki, S.K.: Sensitivity of solar off-limb line profiles to electron density stratification and the velocity distribution anisotropy. Astron. Astrophys. 445(2), 735-745 (2006). https://doi. org/10.1051/0004-6361:20042568

56. Schmelz, J.T., Kimble, J.A., Jenkins, B.S., Worley, B.T., Anderson, D.J., Pathak, S., Saar, S.H.: Atmospheric imaging assembly multithermal loop analysis: first results. Astrophys. J. Lett. 725(1), L34-L37 (2010). https://doi.org/10.1088/2041-8205/725/1/L34

57. Schrijver, C.J., Title, A.M., Hagenaar, H.J., Shine, R.A.: Modeling the distribution of magnetic fluxes in field concentrations in a solar active region. Solar Phys. 175(2), 329-340 (1997). https:// doi.org/10.1023/A:1004901916229

58. Schwenn, R., Marsch, E.: Physics of the inner heliosphere II. Physics and Chemistry in Space 21 (1991) https://doi.org/10.1007/978-3-642-75364-0 
59. Strong, K.T.: YOHKOH Team: Observations of loops and prominences. Space Sci. Rev. 70(1-2), 133-142 (1994). https://doi.org/10.1007/BF00777857

60. Taroyan, Y., Erdélyi, R.: Heating diagnostics with MHD waves. Space Sci. Rev. 149(1-4), 229-254 (2009). https://doi.org/10.1007/s11214-009-9506-9

61. Tomczyk, S., McIntosh, S.W., Keil, S.L., Judge, P.G., Schad, T., Seeley, D.H., Edmondson, J.: Alfvén waves in the Solar Corona. Science 317(5842), 1192 (2007). https://doi.org/10.1126/science. 1143304

62. Toulmonde, M.: The diameter of the Sun over the past three centuries. Astron. Astrophys. 325, 1174-1178 (1997)

63. Tu, C.Y., Marsch, E., Wilhelm, K., Curdt, W.: Ion temperatures in a Solar polar coronal hole observed by SUMER on SOHO. Astrophys. J. 503(1), 475-482 (1998). https://doi.org/10.1086/ 305982

64. Turck-Chièze, S., Carton, P.H., Ballot, J., Barrière, J.C., Daniel-Thomas, P., Delbart, A., Desforges, D., Garcia, R.A., Granelli, R., Mathur, S., Nunio, F., Piret, Y., Pallé, P.L., Jiménez, A.J., JiménezReyes, S.J., Robillot, J.M., Fossat, E., Eff-Darwich, A.M., Gelly, B.: GOLF-NG spectrometer, a space prototype for studying the dynamics of the deep solar interior. Advances in Space Research 38(8), 1812-1818 (2006). https://doi.org/10.1016/j.asr.2005.09.033

65. Verscharen, D., Wicks, R.T., Branduardi-Raymont, G., Erdélyi, R., Frontera, F., Götz, C., Guidorzi, C., Lebouteiller, V., Matthews, S.A., Nicastro, F., Rae, I.J., Retinò, A., Simionescu, A., Soffitta, P., Uttley, P., Wimmer-Schweingruber, R.F.: The Plasma universe: a coherent science theme for voyage 2050. Frontiers in Astronomy and Space Sciences 8, 30 (2021). https://doi.org/10.3389/fspas.2021. 651070

66. Wilhelm, K., Marsch, E., Dwivedi, B.N., Hassler, D.M., Lemaire, P., Gabriel, A.H., Huber, M.C.E.: The Solar Corona above polar coronal holes as seen by SUMER on SOHO. Astrophys. J. 500(2), 1023-1038 (1998). https://doi.org/10.1086/305756

67. Winebarger, A.R., Warren, H.P., Falconer, D.A.: Modeling X-ray loops and EUV Moss in an active region core. Astrophys. J. 676(1), 672-679 (2008). https://doi.org/10.1086/527291

68. Withbroe, G.L.: The temperature structure, mass, and energy flow in the corona and inner solar wind. Astrophys. J. 325, 442 (1988). https://doi.org/10.1086/166015

69. Wittmann, A.D.: Visual and photoelectric measurements of the solar diameter (1972-2002): Methods and results. Astronomische Nachrichten 324(4), 378-380 (2003). https://doi.org/10.1002/asna. 200310135

70. Wittmann, A.D., Bianda, M.: Drift-time measurements of the solar diameter 1990-2000: New limits on constancy. In: Wilson, A. (ed.) The Solar Cycle and Terrestrial Climate, Solar and Space weather, vol. 463, p. 113. ESA Special Publication (2000)

71. Wu, CJ, Usoskin, IG, Krivova, N, Kovaltsov, GA, Baroni, M, Bard, E, Solanki, SK: Solar activity over nine millennia: A consistent multi-proxy reconstruction. Astronomy and Astrophysics in press. arXiv:1804.01302 (2018)

\section{Authors and Affiliations}

Robertus Erdélyi ${ }^{1,6,16}$ ( Luc Damé $^{2} \cdot$ Andrzej Fludra $^{3} \cdot$ Mihalis Mathioudakis $^{4}$. T. Amari ${ }^{5} \cdot$ B. Belucz ${ }^{1,6} \cdot$ F. Berrilli $^{7}$. S. Bogachev ${ }^{8} \cdot$ D. Bolsée ${ }^{9} \cdot$ V. Bothmer ${ }^{10}$. S. Brun ${ }^{11} \cdot$ S. Dewitte ${ }^{12} \cdot$ T. Dudok de Wit ${ }^{13} \cdot$ M. Faurobert ${ }^{14} \cdot$ L. Gizon ${ }^{15}$. N. Gyenge ${ }^{1,16} \cdot$ M. B. Korsós $s^{6,16,17} \cdot$ N. Labrosse ${ }^{18} \cdot$ S. Matthews ${ }^{19}$. M. Meftah ${ }^{2} \cdot$ H. Morgan ${ }^{17} \cdot$ P. Pallé20 ${ }^{20}$ P. Rochus ${ }^{21} \cdot$ E. Rozanov ${ }^{22}$. B. Schmieder ${ }^{23} \cdot$ K. Tsinganos ${ }^{24} \cdot$ E. Verwichte ${ }^{25} \cdot$ S. Zharkov ${ }^{26} \cdot$ F. Zuccarello ${ }^{27}$. R. Wimmer-Schweingruber ${ }^{28}$

Robertus Erdélyi robertus@sheffield.ac.uk

1 School of Mathematics and Statistics, Solar Physics and Space Plasma Research Centre, University of Sheffield, Hicks Building, Hounsfield Road, S3 7RH Sheffield, UK 
2 Laboratoire Atmosphères, Milieux, Observations Spatiales (LATMOS), Guyancourt, France

3 STFC Rutherford Appleton Laboratory, OX11 0QX Chilton, Didcot, UK

4 Astrophysics Research Centre, Queen's University, Belfast, UK

5 CPHT, Ecole Polytechnique, Palaiseau, France

6 Eötvös University, Budapest, Hungary

7 University of Tor Vergata, Roma, Italy

8 Lebedev Physics Institute, Moscow, Russia

9 BIRA-IASB, Brussels, Belgium

10 University of Göttingen, Göttingen, Germany

11 Service d'Astrophysique, CEA Saclay, Saclay, France

12 Royal Meteorological Inst. of Belgium, Brussels, Belgium

13 LPC2E, CNRS/University of Orléans/CNES, Orléans, France

14 IRAP, Université de Nice, Toulouse, LUAN, France

15 Max-Planck-Institut für Sonnensystemforschung, Göttingen, Germany

16 Hungarian Solar Physics Foundation, Gyula, Hungary

17 Aberystwyth University, Wales, UK

18 School of Physics and Astronomy, University of Glasgow, Glasgow, Scotland, UK

19 Mullard Space Science Lab., UCL, Surrey, UK

20 Instituto de Astrofisica de Canarias (IAC), Tenerife, Spain

21 Centre Spatial de Liège, Liège, Belgium

22 PMOD/WRC, Davos, Switzerland

23 Observatory Paris-Meudon, Meudon, France

24 University of Athens, Athens, Greece

25 University of Warwick, Coventry, UK

26 University of Hull, Hull, UK

27 Dipartimento di Fisica e Astronomia, University of Catania, Catania, Italy

28 CAU, University of Kiel, Kiel, Germany 